Pacific Journal of Mathematics

THE ENUMERATION OF HAMILTONIAN POLYGONS IN 


\title{
THE ENUMERATION OF HAMILTONIAN POLYGONS IN TRIANGULAR MAPS
}

\author{
R. C. Mullin
}

A finite nonseparable topological graph $G$ in $E^{2}$ is said to be a triangular map if all its finite faces are triangular. Edges and vertices of $G$ are external if they are incident with the infinite face, otherwise they are internal. The maps considered are rooted by distinguishing an external vertex and incident external edge. A polygon in the graph $G$ of such a map is Hamiltonian if it includes all vertices of $G$. In this paper, the average number of Hamiltonian polygons in a member of the class of nonisomorphic rooted triangular maps with $n$ internal and $m+3$ external vertices is determined. Asymptotic estimates are included for the results obtained. An unexplained coincidence is shown between the number of Hamiltonian polygons in rooted triangular maps and in their duals, rooted nonseparable trivalent maps.

Triangular maps and Hamiltonian polygons. Let $R$ be a simply connected closed region in the Euclidean plane $E^{2}$ whose boundary is a simple closed curve $C$.

A triangular map is a representation of $R$ as the union of a finite number of disjoint point sets called cells, where the cells are of three kinds, vertices, edges and faces (said to be of dimension 0,1 and 2, respectively) where each vertex is a single point, each edge is an open arc whose ends are distinct vertices and each face is a simply connected open region whose boundary consists of the closure of the union of three edges. Two cells of dimension are incident if one is contained in the boundary of the other. Two cells of the same dimension are incident if their closures are not disjoint.

Vertices and edges are external if they are contained in the boundary of $R$. Otherwise they are internal.

A rooted triangular map is a triangular map in which one external vertex is distinguished as the root vertex and an external edge incident with the root vertex is distinguished as the root edge. Two triangular rooted maps $T$ and $T^{*}$ are isomorphic if there exists a bi-unique mapping $f$ of the cells of $T$ onto the cells of $T^{*}$ which preserves dimension and rooting, and both $f$ and $f^{-1}$ preserve incidence.

Isomorphism is clearly an equivalence relation and, as usual, we enumerate only the number of isomorphism classes of such maps. A map is said to be of type $[n, m]$ if it contains precisely $m+3$ external vertices and $n$ internal vertices.

Received April 14, 1964 and in revised form June 4, 1964. 
With every triangular map there is an associated linear graph whose edges and vertices are the edges and vertices of the map and whose incidence relations are those induced by the map. A polygon in a linear graph is a connected di-valent subgraph. If the polygon contains all vertices of the graph, it is called Hamiltonian. In the following we refer to a polygon in the graph of a map as a polygon in the map. A map will be called an $H$-map if it contains at least one Hamiltonian polygon. A Hamiltonian-rooted map is a rooted $H$-map in which a Hamiltonian polygon is distinguished as root polygon. A Hamiltonian polygon $H$ in a map $T$ is said to be internal if no external edge of $T$ is an edge of $H$.

2. Enumeration of Hamiltonian polygons. We enumerate Hamiltonian-rooted maps in terms of the parameter $k=m+3$. We begin with an important special case. A map of type $[0, m]$ will be called a sliced polygon. Every sliced polygon contains exactly one Hamiltonian polygon, namely that consisting of external edges and vertices. It is well-known that there are $\{1 /(k-1)\}\left(\begin{array}{c}2 k-4 \\ k-2\end{array}\right)$ sliced rooted polygons of type $[0, m]$ where $k=m+3$. To determine the number of Hamiltonian-rooted maps and the number of Hamiltonian maps in which the root polygon is internal, we introduce the concept of a $k$-perm.

We define a $k$-perm as an ordered set of $k$ objects, these objects being either directed edge graphs (that is, a graph consisting of a single edge one of whose ends is distinguished as the root) or sliced rooted polygons. We shall show a one-to-one correspondence between

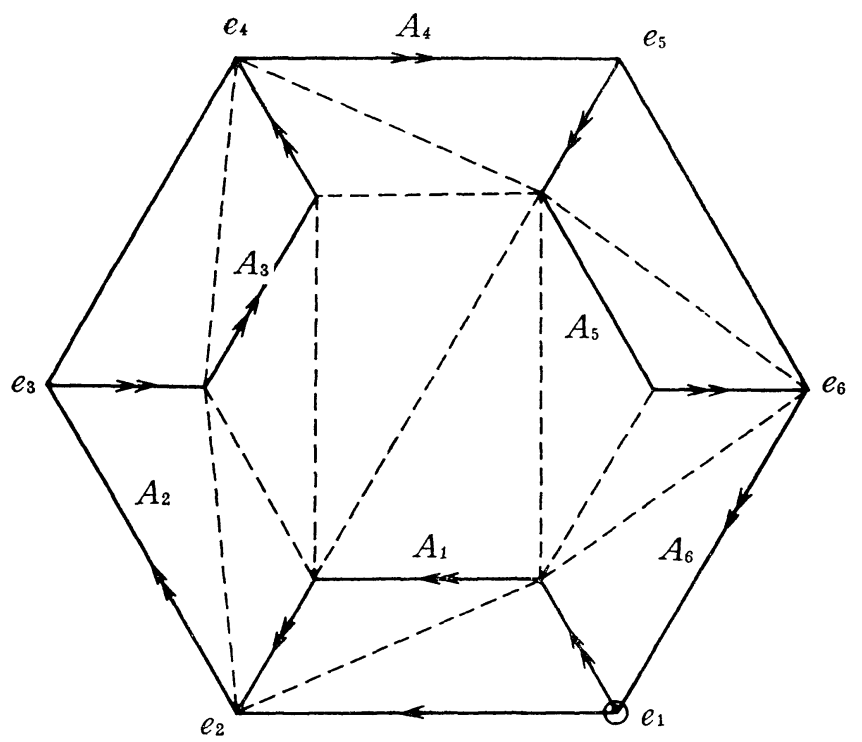

Figure 1 
Hamiltonian-rooted maps and $k$-perm, sliced rooted polygon pairs. Let $H$ be the root polygon of a Hamiltonian-rooted map of type $[n, m]$ $m>0$. Then $H$ contains $n+k$ vertices.

Beginning at the root vertex one can enumerate the vertices of the polygon in one of two orders $S_{1}$ and $S_{2}$. In one of these, say $S_{1}$, the other end of the root edge will be the second external vertex to appear in the sequence, since the external vertices must appear in the Hamiltonian polygon in the same relative orders as they appear in the external polygon. Their order of appearance, of course, depends on the direction in which the polygon $H$ is traversed. (See Figure 1.)

To construct the corresponding $k$-perm, one may proceed as follows. Consider the sequence of vertices $S_{1}$. Label the root vertex $e_{1}$, the other end of the root edge $e_{2}$, and the remaining external vertices as $e_{3}, e_{4}, \cdots, e_{k}$ in accordance with their order of appearance. This defines $k$ arcs of $H, A_{1}=e_{1} e_{2}, A_{2}=e_{2} e_{3}, \cdots, A_{k}=e_{k} e_{1}$. Then in position $j$ in the $k$-perm, $(j=1,2, \cdots, k)$ one constructs an arc $B_{j}$ homeomorphic to $A_{j}$, with ends labelled $b_{j}, b_{j+1}$ being the correspondents of $e_{j}, e_{j+1}$ respectively, the labelling being done modulo $k$. If $B_{j}$ consists of more than one edge, we join $b_{j}$ and $b_{j+1}$ to complete the polygon which is now labelled $B$. Two vertices are joined by a line segment across the interior of the polygonal region bounded by $B_{j}$ if and only if they are joined by an edge interior to the region defined by the correspondent of $B_{j}$ in the original Hamiltonian rooted map. Clearly this produces a sliced polygon in position $j$ of the $k$-perm, this may be rooted by taking the vertex $b_{j}$ and edge $b_{j} b_{j+1}$ as root vertex and edge respectively. This produces a $k$-perm with $k \geqq 2$. To obtain the associated sliced polygon, one selects a polygon $P$ homeomorphic to the Hamiltonian polygon $H$. The vertex corresponding to the root vertex of $H$ is taken as the root vertex of $P$ and the edge defined by the first pair of elements in $S_{1}$ is the root edge of $P$.

Vertices are joined by a line segment across the interior of the polygonal region determined by $P$ if and only if their correspondents are joined across the interior of region bounded by $H$. This produces a rooted sliced polygon. The total number of vertices in the $k$-perm is $2 k+n$. Up to isomorphism, the $k$-perm and polygon pair produced in unique. Evidently if one is given a $k$-perm with $2 n+k$ vertices and sliced rooted polygon with $n+k$ external vertices, one can construct a corresponding Hamiltonian rooted triangular map. We further note that the root polygon of such a map is internal if and only if the corresponding $k$-perm has no elements consisting of a single edge only.

Let $a_{n, k}$ denote the number of $k$-perms which correspond to a Hamiltonian map with $n$ internal vertices. Using the formula for sliced rooted polygons, 


$$
a_{n, k}=\sum_{\alpha_{1}+\alpha_{2}+\cdots+\alpha_{k}=n} \prod_{i=1}^{k}\left(\begin{array}{c}
2 \alpha_{i} \\
\alpha_{i}
\end{array}\right) \frac{1}{\alpha_{i}+1} \text {, where } \alpha_{i} \geqq 0 \text {. }
$$

This is evident if one considers the $k$-perms in which the $i$ th component contains the correspondents of $\alpha_{i}$ internal vertices.

Noting that

$$
\sum_{n=0}^{\infty}\left(\begin{array}{c}
2 n \\
n
\end{array}\right) \frac{1}{n+1} x^{n}=\frac{1-\sqrt{1-4 x}}{2 x}
$$

(which we shall denote by $A(x)$ ) we see that $a_{n, k}$ is the coefficient of $x^{n}$ in the expansion of $A^{k}(x)$.

Noting that $A(x)$ is defined by the equation

$$
A(x)=1+x A^{2}(x),
$$

we employ Lagrange's power series expansion theorem [10, p. 132] to obtain

$$
A^{k}(x)=\sum_{n=0}^{\infty} \frac{k(2 n+k-1) !}{n !(n+k) !} x^{n} .
$$

(There is no ambiguity involved here since (2.2) has only one solution which is analytic at the origin). Hence

$$
a_{n, k}=\frac{k(2 n+k-1) !}{n !(n+k) !}
$$

If $p_{n, k}$ denotes the number of Hamiltonian-rooted maps of type $[n, m]$ then since there are $(2 n+2 k-4) ! /(n+k-1) !(n+k-2)$ ! sliced rooted $n+k-$ polygons to accompany the $k$-perms

$$
p_{n, k}=\frac{k(2 n+2 k-4) !(2 n+k-1) !}{(n+k-1) !(n+k-2) ! n !(n+k) !} .
$$

If we wish to enumerate internally rooted $H$-maps of type $[n, m]$, we must determine the quantity

$$
b_{n, k}=\sum_{\alpha_{1}+\alpha_{2}+\cdots+\alpha_{k}=n} \prod_{i=1}^{k} \frac{1}{\alpha_{i}+1}\left(\begin{array}{c}
2 \alpha_{i} \\
\alpha_{i}
\end{array}\right) \text { where all } \alpha_{i}>0 .
$$

Hence we must find the coefficient of $x^{n}$ in the expansion of

$$
\left[\frac{(1-2 x)-\sqrt{1-4 x}}{2 x}\right]^{k} \text {. }
$$

This author sees no immediately apparent way of obtaining a closed form expression for $b_{n, k}$ by use of the Lagrange formula.

However we note that the differential equation for 


$$
N(x)=\left\{\frac{1-2 x-\sqrt{1-4 x}}{2 x}\right\}^{k}
$$

is

$$
x\left[4 x^{2}-x\right] N^{\prime \prime}(x)+x[6 x-1] N^{\prime}(x)+k^{2} N(x)=0 .
$$

This gives the recursion formula

$$
b_{r, k}=\frac{2(r-1)(2 r-1)}{(r+k)(r-k)} b_{r-1, k},
$$

with $b_{k, k}=1$. Hence

$$
\begin{aligned}
b_{n, k} & =2^{n-k}\left(\begin{array}{c}
n-1 \\
n-k
\end{array}\right) \frac{(2 n-1)(2 n-3) \cdots(2 k-3)}{(k+n)(k+n-1) \cdots(2 k+2)} \\
& =\frac{k}{n}\left(\begin{array}{c}
2 n \\
n+k
\end{array}\right) .
\end{aligned}
$$

If $q_{n, k}$ represents the number of internally rooted $H$-maps of type $[n, m]$, then

$$
q_{n, k}=\frac{k(2 n+2 k-4) !(2 n) !}{n(n+k-1) !(n+k-2) !(k+n) !(n-k) !},
$$

and if $r_{n, k}$ denotes the ratio $q_{n, k} / p_{n, k}$

$$
r_{n, k}=\frac{(2 n) !(n-1) !}{(n-k) !(2 n+k-1) !} .
$$

It is shown in [4] that the number of rooted triangular maps of type $[n, m]$ is

$$
\frac{2^{n+1}(2 m+3) !(3 n+2 m+2) !}{(m+1) !^{2} n !(2 n+2 m+4) !}
$$

Hence the average number of Hamiltonian polygons in a rooted map is

$$
\frac{m+3}{2^{n+1}} \frac{(m+1) !^{2}(2 n+m+2) !(2 n+2 m+2) !(2 n+2 m+4) !}{(2 m+3) !(n+m+1) !(n+m+2) !(n+m+3) !(3 n+2 m+3) !}
$$

Asymptotic formulae. In the following asymptotic approximations, we keep the number of external edges fixed, and observe the behaviour of the formulae as $n \rightarrow \infty$. We find that

$$
p_{n, k} \sim \frac{k}{\pi} 2^{4 n+3 k-5} n^{-3},
$$

and 


$$
q_{n, k} \sim \frac{k}{\pi} 2^{4 n+2 k-4} n^{-3}
$$

Therefore

$$
r_{n, k} \sim \frac{1}{2^{k-1}}
$$

Also the average number of Hamiltonian polygons in a rooted map is asymptotically

$$
\frac{8(m+3)(m+1) !^{2}}{(2 m+3) ! \sqrt{3 \pi}}\left(\frac{32}{7}\right)^{m+1}\left(\frac{32}{27}\right)^{n} n^{-1 / 2}
$$

which increases without bound as $n$ tends to infinity.

3. A coincidence? A rooted trivalent map is the dual of a rooted triangular map in which the external boundary contains three edges. The trivalent map is rooted by calling the edge corresponding the root edge of the triangular map the first major edge, and the edge corresponding to the external edge not incident with the root vertex the second major edge, and the correspondent of the remaining external edge the third major edge. Tutte [6] has shown that if $u_{n}$ denotes the number of rooted trivalent maps in which a Hamiltonian polygon passing through the first and second major edges is distinguished as root polygon, then

$$
u_{n}=\frac{1}{2} \frac{(2 n) !(2 n+2) !}{n !(n+1) !^{2}(n+2) !} .
$$

We note that in this enumeration a restriction is placed on the root Hamiltonian polygon, namely it must pass through the first and second major edges. If we reduce the restriction to merely insist that the Hamiltonian polygon pass through the first major edge, the number of Hamiltonian rooted trivalent maps is

$$
u_{n}^{*}=\frac{(2 n) !(2 n+2) !}{n !(n+1) !^{2}(n+2) !} .
$$

We compare this with the number of Hamiltonian rooted triangular maps of type $[n,-1]$ as given by equation (2.3).

$$
p_{n, 2}=2 \frac{(2 n) !(2 n+1) !}{n !^{2}(n+1) !(n+2) !}=\frac{(2 n) !(2 n+2) !}{n !(n+1) !^{2}(n+2) !}=u_{n}^{*} .
$$

However $u_{n}^{*}$ is the number of Hamiltonian polygons in the class of maps obtained by dualizing the class of rooted triangular maps of type $[n,-1]$; we call these duals "almost trivalent maps" and observe 
that they have $2 n+1$ vertices. Under dualization we understand that the root face of the original map is the root vertex of the dual, etc. Indeed rooted "almost trivalent" map with $2 n+1$ vertices is equivalent to a rooted trivalent map of $2 n$ vertices obtained by considering the divalent root vertex and its incident edges as a single edge, which we take as the first major edge. The remaining end of the root edge is incident with two more edges which are taken as second and third major edge according to a suitable convention, such as the second major edge is adjacent to the root face, whereas the third is not. Clearly every Hamiltonian polygon through the first major edge of the trivalent map is a Hamiltonian polygon in the "almost trivalent" map. Furthermore the above construction is reversible. Hence we conclude that the number of Hamiltonian rooted maps of type $[n,-1]$ is equal to the number of Hamiltonian rooted maps in its dual class, "almost trivalent" maps with $2 n+1$ vertices. But the duals of some of the maps with Hamiltonian polygons contain no Hamiltonian polygons, so that the coincidence is not explained by duality.

The author wishes to thank the referee for his useful suggestions in the revision of this paper.

\section{REFERENCES}

1. W. G. Brown, Enumeration of non-separable planar maps Canad. J. Math. 15 (1963), 526-545.

2. Enumeration of triangulations of the disc, Proc. London Math. Soc. 14 (1964), 746-68.

3. F. Harary, Unsolved problems in the enumeration of graphs, Publications Math. Inst. Hungar. Acad. Sci. 5 (1960), 63-95.

4. R. C. Mullin, The enumeration of rooted triangular maps, Amer. Math. Monthly, 71 (1964), 1007-10.

5. W. T. Tutte, A census of planar triangulations, Canad. J. Math. 14 (1962), 21-38.

6. - A census of Hamiltonian polygons, Canad. J. Math. 14 (1962), 402-417.

7. - A census of slicings, Canad. J. Math. 14 (1962), 708-722.

8. - A census of planar maps Canad. J. Math. 15 (1963), 249-271.

9. - A new branch of enumerative graph theory, Bull. Amer. Math. Soc. 65 (1962), 500-504.

10. E. T. Whittaker and G. N. Watson, $A$ course of modern analysis (Cambridge, 1940).

UNIVERSITY OF WATERLOO 



\section{PACIFIC JOURNAL OF MATHEMATICS}

EDITORS

H. SAMELSON

Stanford University

Stanford, California

R. M. BLUMENTHAL

University of Washington

Seattle, Washington 98105
*J. DUGUNDJI

University of Southern California

Los Angeles, California 90007

RICHARD ARENS

University of California

Los Angeles, California 90024

\section{ASSOCIATE EDITORS}

E. F. BECKENBACH

B. H. NeumanN

F. WOLF

K. YosidA

\section{SUPPORTING INSTITUTIONS}

UNIVERSITY OF BRITISH COLUMBIA

CALIFORNIA INSTITUTE OF TECHNOLOGY

UNIVERSITY OF CALIFORNIA

MONTANA STATE UNIVERSITY

UNIVERSITY OF NEVADA

NEW MEXICO STATE UNIVERSITY

OREGON STATE UNIVERSITY

UNIVERSITY OF OREGON

OSAKA UNIVERSITY

UNIVERSITY OF SOUTHERN CALIFORNIA
STANFORD UNIVERSITY

UNIVERSITY OF TOKYO

UNIVERSITY OF UTAH

WASHINGTON STATE UNIVERSITY

UNIVERSITY OF WASHINGTON

AMERICAN MATHEMATICAL SOCIETY CHEVRON RESEARCH CORPORATION

TRW SYSTEMS

NAVAL ORDNANCE TEST STATION 


\section{Pacific Journal of Mathematics}

\section{Vol. 16, No. $1 \quad$ November, 1966}

Larry Armijo, Minimization of functions having Lipschitz continuous first

partial derivatives ............................... 1

Edward Martin Bolger and William Leonard Harkness, Some

characterizations of exponential-type distributions.............. 5

James Russell Brown, Approximation theorems for Markov operators ...... 13

Doyle Otis Cutler, Quasi-isomorphism for infinite Abelian p-groups ...... 25

Charles M. Glennie, Some identities valid in special Jordan algebras but not valid in all Jordan algebras .......................... 47

Thomas William Hungerford, A description of $\operatorname{Mult}_{i}\left(A^{1}, \cdots, A^{n}\right)$ by

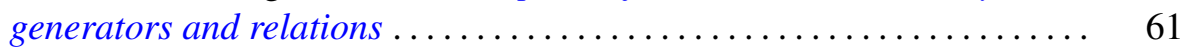

James Henry Jordan, The distribution of cubic and quintic non-residues ....

Junius Colby Kegley, Convexity with respect to Euler-Lagrange differential operators................................... 87

Tilla Weinstein, On the determination of conformal imbedding ......... 113

Paul Jacob Koosis, On the spectral analysis of bounded functions ........ 121

Jean-Pierre Kahane, On the construction of certain bounded continuous functions ................................... 129

V. V. Menon, A theorem on partitions of mass-distribution ........... 133

Ronald C. Mullin, The enumeration of Hamiltonian polygons in triangular

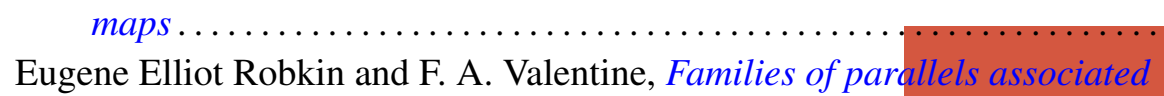

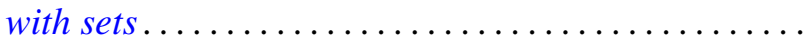

Melvin Rosenfeld, Commutative F-algebras

A. Seidenberg, Derivations and integral closure

S. Verblunsky, On the stability of the set of exponents of a Cauchy

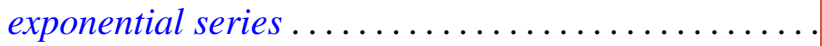

Herbert Walum, Some averages of character sums 